\title{
Características psicopedagógicas y estrategia educativa para escolares con alta capacidad de razonar y aprender en San Ramón, Alajuela, Costa Rica
}

\author{
Zayra Méndez Barrantes ${ }^{1}$ \& Alicia Sandoval Poveda ${ }^{2}$ \\ Universidad Estatal a Distancia, 474-2050 San Pedro Montes de Oca, San José, Costa Rica \\ 'zmendez@uned.ac.cr; 2asandovalp@uned.ac.cr
}

Recibido 30-XI-2010 Corregido 14-II-2011 Aceptado 4-III-2011

\begin{abstract}
Psychopedagogical characteristics and educational strategy for children with high capacities for reasoning and learning in San Ramón, Alajuela, Costa Rica. This study's main objectifs were to identify children with high capacities for reasoning and learning from a constructivist point of view, to describe their psychopedagogical characteristics and to elaborate an educational strategy to attend their needs. Two tests, André Rey's Six Minutes Written Vocabulary and Mathematical Reasoning were applied to fourth grade students of 5 schools belonging to different administrative categories. From a methodological perspective this study is a combination of cuantitative analysis (deciles) and qualitative design (analysis of interviews of children and teachers). Deciles were calculated for each of the groups and for the whole population. The results of the children in these tests were the basis for the identification of the students with higher abilities for reasoning, students situated from decile 8 to 10 were selected. These children were interviewed and asked to make a spontaneous drawing. A guide to observe them in class was given to the teachers, to obtain additional information about them. In order to help the teachers offer them the best learning situations, comprehensible, friendly documents were written on three themes: what is a student with high capacity for reasoning and learning, how to help these students obtain better comprehension in Mathematics and how to stimulate students for better reading and writing abilites. Important conclusions of this study are: $7,07 \%$ of the children were identified as with high learning and reasoning abilites. Among their psychopedagogical characteristics may be named the fact that they do not use reading as a recreative or academic tool and that they do not employ writing or drawing to express their interests in life. Another important result of this study is the fact that it is necessary to train teachers for better identification and academic attention to these children.
\end{abstract}

\section{KEY WORDS}

Learning ability, mathematical reasoning, written vocabulary, decile analysis, talent identification.

\section{RESUMEN}

Los objetivos de este estudio de perspectiva constructivista fueron: identificar niños con alta capacidad para el aprendizaje, describir sus características psicopedagógicas y elaborar una estrategia educativa para atender sus necesidades. En una primera fase cuantitativa se aplicaron la prueba de Vocabulario Escrito de Seis Minutos de Rey (1969) y la de Razonamiento Matemático (Oviedo \& Méndez 1984) a estudiantes de cuarto grado de cinco escuelas. Se calcularon deciles para la población. Los niños situados del decil 8 en adelante en su grupo en ambas pruebas fueron seleccionados como estudiantes de alta capacidad para el aprendizaje. En la segunda fase, de enfoque cualitativo, fueron entrevistados y se les pidió un dibujo espontáneo. Se le entregó a las docentes una guía de observación. Se elaboraron folletos sobre estudiantes de alta capacidad para el aprendizaje, cómo mejorar la comprensión en matemática y estimular las habilidades de lectura y escritura. Un 7,07\% de los niños fueron identificados con habilidades de aprendizaje. Entre sus características psicopedagógicas se encontró que no utilizan la lectura como medio de recreación ni la escritura o el dibujo para expresar sus intereses. Se identificó la necesidad de capacitar a las educadoras en la identificación y atención de estos niños..

\section{PALABRAS CLAVE}

Capacidad de aprendizaje, razonamiento matemático, vocabulario escrito, análisis por deciles, identificación de talento. 
Los estudiantes de alta capacidad o talentosos de acuerdo a Renzulli (2005) se trata de niños con alto potencial para el aprendizaje, creatividad para resolver problemas y motivación para realizar el trabajo. De acuerdo a Sternberg \& Clinkenbeard (1995) hay talento analítico, creativo y práctico. En este proyecto se estudió al alumno de alta capacidad para el aprendizaje y sus características psicopedagógicas.

El interés de esta investigación se basa en el hecho de que si estos niños no son identificados y bien orientados pedagógicamente, su inteligencia se estanca y su comportamiento, con frecuencia, se vuelve agresivo o de total desinterés por los asuntos académicos, presentando una serie de dificultades como resistencia a los métodos de enseñanza, frustración e incomprensión (Castro 2004).

El proyecto surgió a partir de la urgencia de atención a las necesidades educativas de los y las niñas que tienen mayor facilidad para aprender El alumno que no ha sido detectado y por ende no se le ha brindado ningún apoyo en particular, puede generar sentimientos de frustración, baja autoestima, problemas de conducta, dificultades en el rendimiento académico incluso obstáculos en la socialización cotidiana con las y los compañeros y docentes en el aula, con interrupciones frecuentes, ruptura de los planes para clase, construcción de reglas propias y sensibilidad a la crítica (Castro 2004).

No se encuentran, dentro de la programación educativa oficial, estrategias metodológicas, pedagógicas o didácticas que procuren la atención a la diversidad en cuanto a una habilidad superior de aprendizaje. Esto puede ser tan negativo desde un punto de vista psicopedagógico y social para estos niños, como lo sería el no ofrecer el tratamiento que requieren los estudiantes con dificultades para el aprendizaje. El trabajo elaborado en Iberoamérica ha hecho hincapié en la pertinencia de incluir el abordaje del estudiante talentoso en el marco de las necesidades educativas especiales (Castro 2004).

Un caso especial es el que se presenta con los estudiantes talentosos que provienen de grupos en desventaja social. Para trabajar con estas poblaciones debe considerarse el medio en el que se desarrollan y las deficiencias y retos que deben enfrentar (Kelbe 2003).

En todos los estratos sociales hay potencial para el desarrollo del talento, y para detectarlo entra en juego la capacidad del investigador para identificarlo, yendo más allá de las pruebas estandarizadas al trato directo con el niño, la comprensión de su realidad y los informes de los educadores. En particular se recomiendan entrevistas individuales sobre las aspiraciones, pensamientos, percepciones sobre el apoyo de sus padres, conciencia de conocimiento y autodeterminación, así como evaluaciones de la habilidad del estudiante en matemáticas y vocabulario (Borland \& Wright 2004).

Por esta razón, resulta valioso el trabajo para desarrollar estrategias e identificar niños en zonas alejadas del centro y de estratos socioeconómicos bajo y medio bajo, quienes suelen estar lejos de la posibilidad de participar de este tipo de proyectos.

En Costa Rica se han realizado trabajos con objetivos similares en cuanto a la identificación de esta población en el sistema educativo público. Beirute \& De Bravo (1984) localizaron que hay un $6 \%$ de estudiantes con talento.

El antecedente principal del actual estudio es el estudio realizado en la Región de Turrialba sobre el proceso de aprendizaje de niños talentosos (Méndez et al. 1992).

En este estudio participativo, interinstitucional e interdisciplinario, se emplearon dos muestras de sujetos. La primera se refería al conjunto de alumnos que atendían los veinticinco educadores que participaron en el estudio. La segunda, fue una submuestra constituida por sesenta niños considerados talentosos por sus maestros, mediante el Cuestionario a docentes para la identificación de estudiantes con altas capacidades (Méndez et al. 1992).

En este estudio se puso en evidencia la existencia de un grupo de niños talentosos en la Región de Turrialba. De la muestra de cuatrocientos setenta y cuatro estudiantes, se identificaron sesenta niños como talentosos, lo que significó un $12,6 \%$ de la muestra total. Sin embargo, la población siguió sin atención al no obtener respuesta del Ministerio de Educación tras presentar los resultados.

Por otra parte desde la Universidad Estatal a Distancia se ha desarrollado desde el 2005 el trabajo en la temática de los niños superdotados. En primer lugar se trabajó con los docentes, utilizando una entrevista de opinión docente para investigar el concepto que manejaban sobre la superdotación y las necesidades educativas especiales asociadas a esta. A partir de este estudio, concluyó que aunque la opinión de los docentes sobre algunas de las características de los estudiantes superdotados coinciden con la teoría y los expertos, también mantienen mitos sobre estos, como que todos deben tener un brillante rendimiento académico. Además, se encontró poca capacitación en los docentes entrevistados sobre el tema de superdotación y que estos están conscientes de que esta población tiene necesidades educativas especiales a las que no se está dando respuesta, aunque consideran que pueden integrarse al sistema educativo (Morales 2009).

Además, se realizó la macroinvestigación de identificación temprana con base empírica de niños con superdotación entre los 4 y los 6 años de edad, llevando acabo la validación en Costa Rica. Se realizó por medio de un Test de 
screening con los padres de familia en centros educativos de nivel materno infantil en San José, Alajuela, Cartago, Guanacaste y Limón. A los niños con resultados positivos se les aplicó el WIPSSI y posteriormente la escala de color del test Raven. Con esta metodología se identificó de la muestra total de 611 estudiantes a un 2,29\% con superdotación, con una mayor cantidad de mujeres y de alumnos procedentes de zonas rurales (Morales 2008).

A pesar de que estos estudios realizados en el país han puesto en evidencia la presencia de niños con alta capacidad para el aprendizaje, no se ha llevado a cabo todavía un plan general que los tome en cuenta para orientarlos en sus aprendizajes escolares y en especial, motivarlos mejor para el estudio y para que alcancen mayores logros en sus vidas personales.

Esta fue una de las razones por las que se pensó en volver a plantear un estudio que identificara niños talentosos según criterio geográfico (Smutny \& Lind 2003), esta vez en la Región Educativa de San Ramón de Alajuela, con apoyo del Consejo Nacional de Rectores (CONARE) y la Universidad Estatal a Distancia (UNED).

Esta investigación tuvo por objetivos generales, en primer lugar contribuir al proceso de identificación de estudiantes con altas capacidades en la región educativa de San Ramón; en segundo, describir las características psicopedagógicas de estos estudiantes y en tercer lugar elaborar conjuntamente con los educadores un plan de atención a los niños talentosos.

\section{METODOLOGÍA}

Se abordó el objetivo de investigación con un enfoque mixto definido temporalmente en dos fases, la primera de ellas desarrollada en el 2009 y la segunda en la primera mitad del 2010.

El trabajo se desarrolló en el circuito 01 de la región educativa de San Ramón. Se utilizó un muestreo por conveniencia (Hernández 2009) para elegir las escuelas en las cuales fuera posible realizar el trabajo de acuerdo a lo dispuesto por el Ministerio de Educación Pública. Se determinó previamente que se tratara de cinco escuelas públicas de dirección administrativa diferente: Dirección 4, Dirección 3, Dirección 2, Dirección 1 y Unidocente.

\section{Fase I}

La primera fase fue de corte cuantitativo. La muestra inicial constó de 212 estudiantes, 127 hombres y 95 mujeres, de los grupos de cuarto grado de las cinco escuelas participantes. Sin embargo, sólo a 139 estudiantes se les pudo aplicar todas las pruebas. Se evaluó a los estudiantes de cuarto grado de cada una de las escuelas, contando con la autorización de la dirección regional del Ministerio de educación y los encargados de cada centro de enseñanza.

En la primera fase, los instrumentos aplicados a toda la muestra fueron:

- Prueba de Vocabulario Escrito en Seis Minutos (Rey 1969, Méndez et al. 1992): se utiliza para evaluar cantidad de vocabulario, con una fase posterior de análisis cualitativo de este (tipos de palabras, agrupaciones, ortografía). Consiste en que cada estudiante escribe en una hoja todas las palabras que desee durante un intervalo de seis minutos, los cuales son medidos con cronómetro. Posteriormente se cuenta el número de palabras escritas por cada niño, y el número del total de palabras, independientemente de la ortografía, es el resultado de la prueba.

- Prueba de Razonamiento Matemático (Oviedo \& Méndez 1984; basada en Vergnaud 1981, Méndez et al. 1992): evalúa la capacidad para resolver problemas aditivos. Es una prueba de ocho ítemes con problemas que evalúan el manejo adecuado de transformaciones como ganar y perder, entrar y salir. Fue adaptado y validado en Costa Rica por Oviedo \& Méndez (1984) en el Área Metropolitana seleccionando los ítemes más complejos de la prueba original de Vergnaud (1981). Cada problema se puede calificar con dos, uno o cero puntos para una puntuación total de 16 . Un punto equivale a la respuesta correcta de cantidad y otro a que el estudiante señale el tipo de transformación que predomina (gana, pierde, entran más, salen más).

- Cuestionario a docentes para la identificación de estudiantes con altas capacidades (Mendez et al. 1992): tiene 26 afirmaciones relativas a los estudiantes sobre su conducta en el aula, las cuales las docentes deben asociar con los nombres de los alumnos que a su parecer las cumplen. Se cuenta cuántas veces aparece cada estudiante mencionado por la maestra, para catalogarlos luego en un ranking de acuerdo al número de menciones obtenidas. El cuestionario puede verse en el Apéndice 1.

Se calificó cada prueba, obteniendo una lista de resultados de la prueba de Vocabulario de Seis Minutos con la cantidad de palabras escrita por cada estudiante, y una lista de resultados de la prueba de Razonamiento Matemático con los puntos obtenidos por los estudiantes.

La habilidad para la matemática se conecta con la memoria y la rapidez, mientras que la habilidad verbal se relaciona con el manejo de conocimiento, por lo que un buen desempeño en cada área señala habilidades cognitivas 
distintas (Benbow \& Minor 1990). Por esta razón se eligieron dos pruebas donde cada una evaluara un aspecto de cada una de estas habilidades.

Posteriormente, se elaboraron las escalas de deciles para cada lista, clasificando a los niños. Los deciles son un caso particular de percentil, se distribuyen todos los datos de menor a mayor y posteriormente se divide en diez partes iguales, de manera que los datos de cada parte son mayores a las que le anteceden y menores a las que le siguen. Son un valor de posición que permite establecer en dónde se encuentra un dato con respecto a los otros (Gómez 2009). Se utilizó esta medida con el objetivo de comparar a los estudiantes con su propio grupo, en lugar de utilizar criterios normativos ajenos a su ambiente.

Por esta razón, una vez establecidos los deciles, se compararon las listas de los estudiantes cuyos resultados se encontraban ubicados del decil 8 en adelante, decil que implica que el estudiante tuvo un desempeño que sólo un 20\% o menos de su grupo alcanzó.

Se definió como niño de alta capacidad para el aprendizaje al alumno que se situó por encima del decil 8 en las dos pruebas aplicadas, entendiendo por tanto que tenía un rendimiento superior al $80 \%$ de sus compañeros en ambas pruebas.

Una vez seleccionados, se analizaron los cuestionarios de las docentes para verificar si los estudiantes destacados en las pruebas eran también los identificados por ellas.

\section{Fase II}

La segunda fase fue de corte cualitativo. Tras la identificación de los estudiantes de alta capacidad para el aprendizaje, se ubicaron 15 de la muestra total $(\mathrm{N}=212)$, pero 6 de ellos se encontraban para el 2010 en escuelas ajenas al estudio. Por esta razón, en la segunda fase se trabajó con 9 alumnos, 5 niños y 4 niñas, identificados como estudiantes de alta capacidad para el aprendizaje que continuaban en las escuelas. No se pretendía generalizar resultados a partir de lo encontrado con estos estudiantes, sino conocer al grupo identificado.

Tras brindar un primer informe a las escuelas sobre los resultados obtenidos y retomar contacto al inicio del nuevo curso lectivo del 2010, se procedió a realizar un estudio de casos con los niños identificados y sus docentes, ahora en quinto grado. Los instrumentos aplicados en esta etapa fueron:

- Entrevista realizada a los niños identificados como talentosos: consiste en una entrevista semi-estructurada con cuatro secciones: relación con la escuela y preferencias académicas (materias de mayor y menor interés, mejores notas, notas más bajas, conducta y relación con los compañeros), la familia (con quienes vive, quienes le ayudan a estudiar), gustos y preferencias (lectura, escritura y tiempo libre) y sus deseos de estudio en el futuro (oficios y profesiones que les gustaría ejercer). La información obtenida se clasificó en esas cuatro categorías, para la descripción de cada caso.

- Dibujo espontáneo: consiste en entregarle a cada estudiante una hoja en blanco y un lápiz, y se le da como instrucción "dibuja lo que quieras". Por medio del dibujo se buscaba evaluar la creatividad. No se trata de una prueba estandarizada de creatividad, sino que se realiza un análisis cualitativo de las categorías: madurez psicomotora, la capacidad de expresar intereses o emociones, originalidad, uso del espacio en el papel, proporcionalidad de los elementos y representación de la tercera dimensión. No se pretende dar un resultado de "nivel" de creatividad, sino caracterizar el desempeño del niño. Estas categorías se respaldan en los criterios de autores que han estudiado el desarrollo del dibujo en los niños (Lowenfeld \& Lambert 1973)

- Instrumento de percepción de las maestras del estudiante de alta capacidad: se trata de un cuestionario informativo diseñado con la información que se necesitaba recabar de cada estudiante, para asegurarse de obtener los mismos datos sobre todos, diseñado para obtener insumos de la observación diaria de las educadoras sobre el comportamiento de los estudiantes escogidos. En este, se recaban los datos del estudiante a evaluar, para posteriormente responder a una escala Likert de los comportamientos del niño en el aula (creatividad, liderazgo, motivación, vocabulario, rapidez de aprendizaje, atención, participación y responsabilidad) y preguntas abiertas sobre la apreciación de la maestra (interés de los padres, habilidades especiales, problemas de aprendizaje y opinión general). La información obtenida en cada caso se resumió clasificando las actitudes de los estudiantes según la frecuencia observada por las maestras y anotando las dificultades identificadas por estas en cada estudiante, para sumarlas a los datos recabados de su caracterización. Puede consultarse en el Apéndice 2.

Se realizó un análisis de caso para cada uno de los estudiantes, teniendo como base la entrevista realizada, las observaciones de las maestras y el resultado de los dibujos. Como insumo extra se realizó un análisis cualitativo de la prueba de vocabulario de los estudiantes destacados, para determinar el tipo de palabras utilizadas, así como las estrategias usadas para obtener un mayor número y la ortografía. 
Una vez con cada caso resumido, se compararon los siguientes aspectos: materias de interés, notas mayores y menores, características familiares, actitud hacia la lectura, la escritura y el tiempo libre, planes de futuro, resultados del dibujo y análisis cualitativo del vocabulario. A esto se sumó la observación de cada educadora. A partir de esta información se realizó la caracterización de los estudiantes identificados.

\section{Trabajo con las educadoras}

A lo largo de la realización de la segunda fase, se hicieron reuniones con las maestras donde se presentaban los resultados obtenidos y se comentaba sobre el desempeño de los niños identificados.

Esto junto a lo comunicado por los niños en las entrevistas llevó a la elaboración de un plan para ayudar a las educadoras a brindar a los niños identificados mayores oportunidades académicas y una atención más individualizada; a cada educadora se le brindó una serie de folletos con temas de interés para atender mejor a los niños y un apoyo personalizado ofrecido por las investigadoras del proyecto cuando visitaban las escuelas.

\section{RESULTADOS}

A continuación se presentan los resultados obtenidos en cada etapa de la investigación. En primer lugar, los resultados de las pruebas para la población general, y en segundo la caracterización de los estudiantes identificados como de alta capacidad para el aprendizaje.

\section{Resultados de la primera fase del estudio}

En la prueba de vocabulario el promedio de palabras escritas fue de $48(\mathrm{~N}=192)$, con más de 63 palabras escritas por los estudiantes ubicados del decil 8 en adelante (Cuadro 1).

Los estudiantes de la escuela de Dirección 4 obtuvieron una puntuación promedio en vocabulario por encima de la media de la población con 51 palabras. Los alumnos de la escuela de Dirección 3 tuvieron la puntuación promedio más baja en vocabulario escrito con 38 palabras, mientras que los niños de la escuela de Dirección 2 tuvieron el promedio más alto de vocabulario escrito con 55 palabras. Por su parte, los estudiantes de la escuela de Dirección 1 se mantuvieron cerca de la puntuación promedio de vocabulario con 49 palabras, y los alumnos de la escuela unidocente tuvieron una puntuación promedio de 46 en esta prueba (Fig. 1).

En la prueba de razonamiento matemático el promedio de puntos alcanzados fue de $5(\mathrm{~N}=159)$ de los 16 puntos posibles (Cuadro 2).

La escuela de Dirección 4 mantuvo la misma puntuación promedio que la población general, al igual que la de Dirección 3 y Dirección 1. La escuela de Dirección 2 tuvo el promedio más bajo con tan solo 3 puntos, mientras que la escuela Unidocente presentó el promedio más alto de puntos obtenidos con 7 (Fig. 2).

\section{CUADRO 1}

Deciles para la población general en la prueba de "Vocabulario de seis minutos"

\begin{tabular}{cc}
\hline Deciles & Puntuación \\
\hline 1 & 25 \\
2 & 33 \\
3 & 38 \\
4 & 43 \\
5 & 48 \\
6 & 52 \\
7 & 57 \\
8 & 63 \\
9 & 70 \\
10 & 91
\end{tabular}

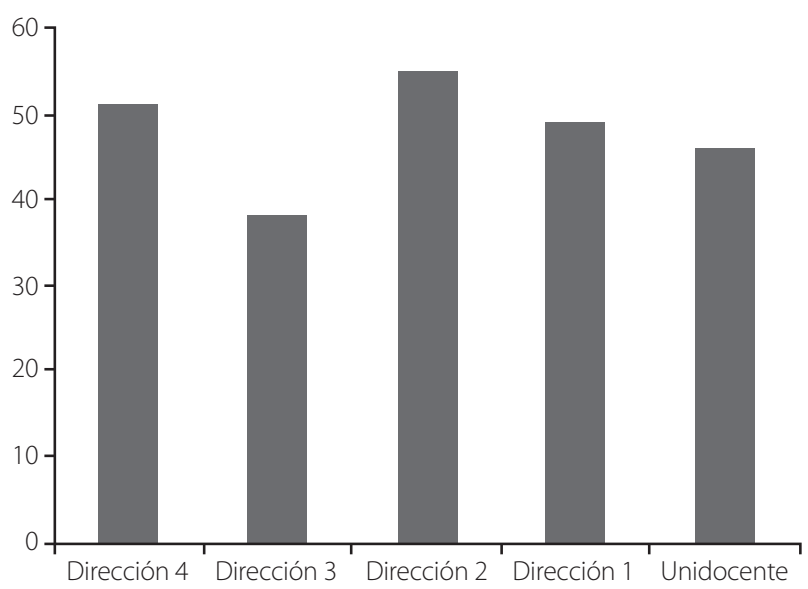

FIG. 1. Promedio de palabras obtenidas en la prueba de "Vocabulario de seis minutos" en cada una de las direcciones. 
CUADRO 2

Deciles para la población general en la prueba de "Razonamiento matemático"

\begin{tabular}{cc}
\hline Deciles & Puntuación \\
\hline 1 & 1 \\
2 & 2 \\
3 & 3 \\
4 & 4 \\
5 & 5 \\
6 & 6 \\
7 & 6 \\
8 & 7 \\
9 & 9 \\
10 & 15
\end{tabular}

En cuanto al cuestionario a docentes para la identificación de estudiantes de alta capacidad, se encontró que los estudiantes que mencionaban no se encontraban entre los destacados en ambas pruebas. De los 15 estudiantes que se ubicaron del decil 8 en adelante, solo 3 fueron mencionados por las educadoras.

Sin embargo, de las veintiséis cualidades evaluadas en el cuestionario como características relacionadas con algún comportamiento en el aula que se relacionaría con la capacidad para el aprendizaje, una docente de la escuela de dirección 4 encontró seis relacionadas con uno de sus estudiantes que posteriormente fue identificado como de alta capacidad para el aprendizaje. A otra niña de las identificadas en la escuela de dirección 4 su docente le identificó una de las cualidades. Finalmente, al estudiante seleccionado de la escuela de dirección 1 la educadora le atribuyó tres de las características evaluadas. Sólo el $20 \%$ de los estudiantes identificados en el estudio fueron señalados por sus docentes.

Los niños identificados se distribuyeron entre cuatro de las escuelas participantes. En la escuela unidocente no se encontró a ninguno destacado por encima del $80 \%$ de su grupo en ambas pruebas. En la escuela de dirección 4 se encontraron once niños, en la dirección 3 y la dirección 1 se encontró un estudiante en cada una, mientras que en la de dirección 2 se encontraron dos estudiantes.

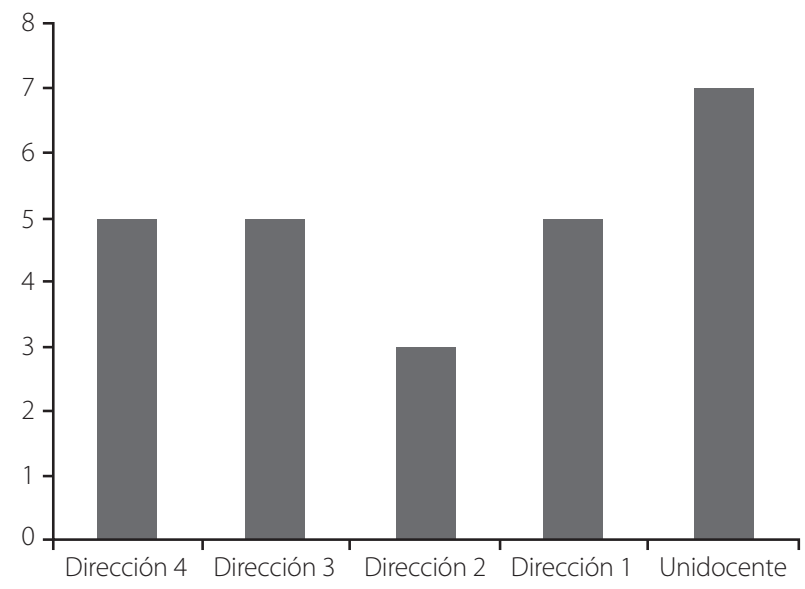

FIG. 2. Promedio de puntos obtenidos en la prueba de "Razonamiento matemático" en cada una de las direcciones.

\section{Resultados obtenidos con la muestra de los estudiantes de alta capacidad para el aprendizaje en la segunda fase del estudio}

En total 15 estudiantes ( 8 mujeres y 7 hombres) se ubicaron en del decil 8 de ambas pruebas, lo que supone un $7,07 \%$ de la muestra total que participó en la investigación. De ellos, solo tres habían sido identificados por las maestras en el cuestionario a docentes para la identificación de estudiantes con altas capacidades.

Al iniciar en el 2010 la etapa de estudio cualitativo con los estudiantes destacados, 6 de los niños habían sido trasladados a escuelas que no participaban en el estudio. Por esta razón se trabajó con 9 alumnos, 4 niñas y 5 niños (uno de los cuales se trasladó a mitad del primer trimestre).

La distribución de estudiantes identificados no fue igual entre las escuelas. En la escuela unidocente ningún alumno superó el decil 8 en ambas pruebas, mientras que en la escuela de Dirección 4, 6 estudiantes alcanzaron esta condición. Se identificó uno en cada una de las escuelas restantes.

Por medio de la entrevista y las observaciones recabadas de las educadoras fue posible determinar algunas características de los estudiantes con los cuales se trabajó. En cuanto a las características familiares, 6 vienen de hogares integrados y 3 se están criando solo con la mamá, ninguno es hijo único. Solo uno de los niños proviene de una familia dedicada a la actividad agropecuaria, y todos son de un estrato socioeconómico medio-bajo. Sobre su tiempo libre la mayoría se dedica a actividades deportivas y de interacción con otros. 
El grupo de estudiantes identificados se dividió en intereses por las materias, la mitad mostró inclinación por las matemáticas y la otra mitad por el español. Ciencias parece ser la que menos les interesa, seguida de estudios sociales. No se encontraron problemas importantes de conducta. En la entrevista se indagaron también sus planes de futuro. Dos de ellos dijeron no tener planes, tres se refirieron a carreras profesionales, dos a oficios manuales y otros dos casos contemplaron ambas posibilidades.

A pesar de que ocho de los estudiantes refirieron que les gusta leer, fue posible apreciar a través de las entrevistas realizadas que la mayoría no ve la lectura como una actividad más allá del ámbito escolar. De igual forma, la escritura y el dibujo se presentan como actividades académicas, sólo dos estudiantes mencionan la escritura como medio de expresión.

Además, en el análisis cualitativo de la prueba de vocabulario de los estudiantes se encontró que el vocabulario usado es esencialmente concreto y conformado de sustantivos, sólo 2 niñas utilizaron menos de un $90 \%$ de sustantivos en su listado de palabras.

En el dibujo espontáneo fue posible apreciar los criterios establecidos de evaluación, todos características a evaluar en el desarrollo de la capacidad creadora (Lowenfeld \& Lambert 1973).
En la mayoría, se observa un buen uso del espacio en el papel y adecuada proporcionalidad en el tamaño de los distintos elementos representados; sin embargo, se aprecia, también, la presencia de elementos estereotipados propios de dibujos hechos por niños de menor edad, así como falta de representación de la tercera dimensión. Sólo en cuatro estudiantes el dibujo tiene alguna relación con posibles intereses personales; en los demás, la representación hecha no parece corresponder a una expresión de intereses propios del alumno. Estas características son más propias de los niños de menor edad (Lowenfeld \& Lambert 1973). En la Fig. 3 se inserta un dibujo realizado por una de las niñas identificadas como de alta capacidad para el aprendizaje, el cual ilustra lo acá encontrado.

\section{Plan de trabajo con las educadoras}

Se optó por un método que a pesar de los frecuentes cambios de maestras, pudiera alcanzar a todas las educadoras por igual y que las nuevas pudieran retomar con facilidad el plan de trabajo con los niños.

Por esta razón se decidió formular folletos dirigidos a las educadoras, el primero de ellos con contenido especializado en niños talentosos y dos más con estrategias constructivistas de enseñanza que podían resultar en

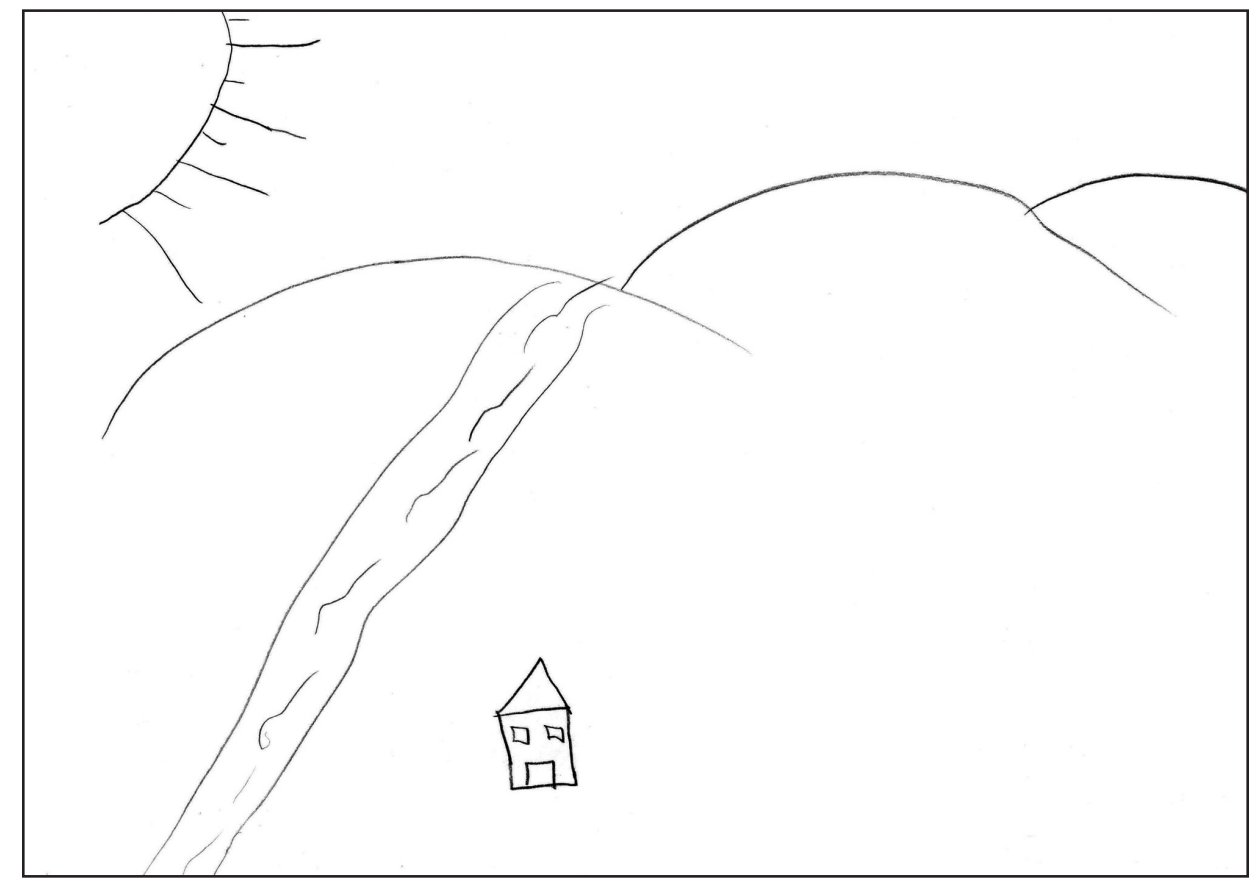

FIG. 3. Dibujo realizado por una estudiante identificada en la escuela de Dirección 4. 
beneficio de todo el grupo, y a su vez favorecer a los estudiantes talentosos. Los folletos fueron:

- Intervenciones con niños talentosos: contiene recomendaciones generales para trabajar con los estudiantes de estas características. Se explicaron los tres tipos de intervenciones con niños talentosos (aceleración y flexibilización, agrupamiento y enriquecimiento curricular), y se desarrollaron cuatro ejes: atención individualizada, participación del estudiante en actividades distintas, papel activo en el aula y creatividad.

- Sugerencias para desarrollar la comprensión en matemática: se basó en la teoría de Piaget, explorando las definiciones de conocimiento físico, conocimiento lógico matemático y conocimiento social o convencional, así como conocimiento conceptual y de procedimiento. A partir de los planteamientos de Van de Walle (1993) se desarrollaron sugerencias para mejorar la comprensión de relaciones matemáticas en los niños y una guía para evaluar las actividades que plantean en clase. Además se añadieron sugerencias de juegos matemáticos tomadas del Centro Nacional de Ciencia y Tecnología (2001a, 2001b) y Méndez \& Pereira (1985).

- Sugerencias para estimular la lectura y la escritura de los niños: se desarrolló para motivar la lectura en los estudiantes, incluyendo aspectos necesarios para favorecer el gusto por la lectura, tomando sugerencias de Ferreiro (2008) y Leal (2003). Posteriormente, se expuso el sistema de lectura creadora según Massip (2004), para impulsar la lectura crítica y creativa. En cuanto a la escritura, se tomaron en cuenta las sugerencias de Quirós (2000) para motivarla.

\section{DISCUSIÓN}

Un 7,07\% de la muestra total son niños que se identificaron como de alta capacidad para el aprendizaje y obtuvieron un resultado por encima de un decil 8 en las pruebas empleadas para seleccionarlos. Identificar una importante proporción de niños de alta capacidad para el aprendizaje en la Región Educativa de San Ramón, alejada de San José y con estudiantes que en su mayoría provienen de estratos socioeconómicos medio y medio bajo, es importante tanto para la sociedad costarricense como para las autoridades del Ministerio de Educación Pública y las Universidades formadoras de maestros y profesores.

De esta manera, se coincide con los resultados de Méndez et al. (1992), Beirute \& De Bravo (1984) y Morales (2008) en confirmar la existencia de niños con alta capacidad para el aprendizaje en el sistema educativo costarricense que no han sido identificados con anterioridad.
En cuanto a las características de los estudiantes identificados, son varias las que se han tomado en consideración, estudiando a los niños en su contexto familiar y comunal.

La representación de género en la muestra de niños con alta capacidad es semejante para los varones y las niñas. De los quince niños originalmente detectados, 7 son hombres y 8 son mujeres. Este es un dato que debe considerarse, ya que la identificación temprana de la alta capacidad para el aprendizaje en las niñas puede ayudar a evitar las limitaciones sociales a la estimulación del talento femenino señaladas por Valadez (2004).

Los niños identificados provienen de familias de estrato medio a medio bajo, lo que puede relacionarse con la propuesta de Borland \& Wright (2004) de que es posible encontrar talento en los distintos estratos sociales.

En su mayoría los estudiantes identificados son de familias integradas. Solo uno de los padres de los niños entrevistados se dedica a labores agrícolas, los demás son comerciantes, policías o se dedican a otros oficios. Las madres se dedican a labores manuales sencillas. Esto podría relacionarse con que entre las carreras y profesiones de interés por parte de los menores no se encuentre ninguna de índole agrícola, lo que llama la atención por tratarse el lugar del estudio de una zona con predominancia de actividades agrícolas y ganaderas (Jiménez 1997).

En cuanto a los intereses de los niños por materias escolares, se encontró inclinación hacia la matemática o el español en la mayoría de los casos, coincidiendo con aquellas en las que tienen un mejor desempeño. Se encontró una tendencia de los niños por identificar las actividades que se realizan en la escuela como algo meramente académico, como es el caso de la lectura, la escritura y el dibujo, los cuales no parecen estar funcionando como actividades recreativas ni como medios de expresión, ni siendo estimuladas en este sentido.

Parece ser por tanto, siguiendo la clasificación de talento de Sternberg y Clinkenbeard (1995) que el talento que se destacó en los casos estudiados, fue el analítico, relacionado con la capacidad de aprender, comparar, analizar, evaluar y juzgar; probablemente el más estimulado por el medio educativo, sin embargo el talento práctico señalado por la habilidad para desenvolverse en el mundo social podría verse reflejado en la tendencia del grupo de niños entrevistados a actividades de interacción con otros, aunque de acuerdo a la observación de las docentes no muestren iniciativas de liderazgo.

El trabajo con las educadoras permitió apreciar que en la mayoría de los casos no estaban de acuerdo con que los estudiantes identificados tuvieran realmente alta capaciad para el aprendizaje. Parece ser que para ellas este estudiante sería el que da buen rendimiento en el aula, se 
comporta bien, cumple con las tareas y es, además, líder de su grupo.

La capacidad de liderazgo es considerada por muchas de estas educadoras, como un rasgo distintivo de un niño talentoso. Aunque algunos autores lo mencionan como una característica a considerar (Ross 1993, citado por Reis 2004), otros autores señalan que hay varios tipos de talento con características diferentes, y todos deben ser considerados respondiendo a necesidades diferentes (Benbow \& Minor 1990, Sternberg \& Clickenbeard 1995, Renzulli 2008).

Fue posible percibir la necesidad de capacitar a las educadoras en el tema de los niños de alta capacidad para que puedan identificarlos, sin confundir el talento con el desempeño académico, o la falta de éste con la hiperactividad o dificultades de concentración.

En distintos estudios los autores señalan la necesidad de tener educadores altamente preparados para atender a estos estudiantes (Mirman 2003) y cómo el éxito de las intervenciones depende de la calidad de los educadores que trabajen con ellos, en cuanto a su preparación y su actitud para abordar el trabajo con el niño de alta capacidad (Kelbe 2003). Por tanto, la clarificación de conceptos y modificación de ideas preconcebidas sobre el talento, así como apoyar a las educadoras en la generación de estrategias de aprendizaje más creativas e innovadoras, repercutiría positivamente en los estudiantes en general.

Por esta razón el primero de los documentos desarrollados para las educadoras se centró en el tema del talento. Los siguientes dos folletos se enfocaron en estrategias generales para mejorar la comprensión en los estudiantes de la matemática y estimular la lectura y escritura. Estos folletos tenían un enfoque más global, dirigido a su aplicación con todo el grupo, siguiendo la línea de Renzulli (2008) de integrar al programa de todos los estudiantes las estrategias para mejorar el rendimiento de los niños de alta capacidad, permitiendo a todos los alumnos mejorar sus destrezas de pensamiento superior, trabajando así desde un modelo de enriquecimiento escolar. Este tiene por objetivo que todos los estudiantes tengan las oportunidades, recursos y refuerzos para maximizar su potencial.

Tras el análisis realizado se llegó a las siguientes conclusiones principales:

1. Se identificó un $7,07 \%$ de estudiantes de alta capacidad para el aprendizaje en la población estudiada. Hay una representación semejante de ambos sexos entre los estudiantes identificados.

2. Se trata de un grupo de alumnos que provienen del estrato económico medio y medio bajo, provenientes de hogares integrados con al menos un hermano, sin hábito de lectura, escritura o dibujo como medio de entretención y/o expresión. Parecen tener un vocabulario concreto, presentan un rendimiento académico promedio, no destacado, con predilección por las matemáticas o el español pero poco interés demostrado en las ciencias.

3. Las docentes necesitan una mayor capacitación en el tema de la alta capacidad para el aprendizaje, la identificación y trato con estos estudiantes.

Es necesario que en Costa Rica se desarrolle la intervención con niños de alta capacidad para el aprendizaje. En Iberoamérica el desarrollo práctico, más allá de la identificación y las propuestas, ha sido poco. En general se han aplicado técnicas de aceleración -adelantar a los niños de nivel escolar- y de enriquecimiento en el aula. Sin embargo, la carencia de formación en los docentes sobre la identificación y trabajo con los niños talentosos está presente en general en la zona (Castro 2004).

Un abordaje adecuado de los niños de alta capacidad para el aprendizaje puede representar un valioso aporte a la evolución económica y social de Costa Rica. El Ministerio de Educación favorece los aprendizajes escolares de estudiantes que se encuentran por debajo del promedio, los cuales son atendidos por educadores especiales y otros especialistas educativos, pero no ha previsto ofrecer al alumno con alta capacidad para el aprendizaje una atención particularizada.

Por otra parte, las Universidades donde estudian los futuros maestros y profesores no tienen aún un gran desarrollo en identificar y atender adecuadamente a los niños con altas capacidades para los aprendizajes, aunque en distintos estudios realizados en Costa Rica desde los años 80 hasta la actualidad, se ha puesto en evidencia la presencia de estos niños en las escuelas de distintas regiones del país (Beirute \& De Bravo 1984, Méndez et al. 1992, Morales 2008).

Costa Rica no se puede permitir el no identificar, guiar y apoyar a estos niños y niñas que tienen el potencial para llegar a ser ciudadanos de provecho, que puedan dar aportes importantes al desarrollo social y económico de nuestro país.

\section{AGRADECIMIENTOS}

Se agradece a la Vicerrectoría de Investigación y el Sistema de Estudios de Posgrado de la Universidad Estatal a Distancia, así como a CONARE (Consejo Nacional de Rectores de Costa Rica) por su apoyo económico y administrativo a este proyecto. De igual forma, se agradece al Ministerio de Educación en San Ramón por la autorización para 
trabajar en la zona y el acceso para visitar las escuelas del proyecto e interactuar con los niños y docentes. Finalmente, se agradece a las directoras y docentes de cada una de las escuelas del proyecto por su permiso para ser visitadas y su apertura en la aplicación de pruebas y entrevistas con las maestras y los niños.

\section{REFERENCIAS}

Beirute, L. \& O. De Bravo. 1984. El niño talentoso en Costa Rica: la adecuación de un currículum para el fomento de su creatividad en el aula regular. Universidad de Costa Rica, San José, Costa Rica.

Benbow, C. \& L. Minor. 1990. Cognitive Profiles of Verbally and Mathematically Precocious Students: Implications for Identification of the Gifted, p. 87-99, In J. Renzulli (ed.). Identification of Students for Gifted and Talented Programs. Corwin, California, Estados Unidos.

Borland, J. \& L. Wright. 2004. Identifying Young, Potentially Gifted, Economically Disadvantaged Students, p. 25-41, In J. Renzulli (ed.). Identification of Students for Gifted and Talented Programs. Corwin, California, Estados Unidos.

Castro, E. 2004. Perspectivas futuras de la educación del niño con talento, p. 171-185, In M. Benavides, A. Maz, E. Castro \& R. Blanco (eds.). La educación de niños con talento en Iberoamérica. Oficina Regional de Educación para América Latina y el Caribe. UNESCO, Santiago, Chile.

Centro Nacional de Ciencia y Tecnología. 2001a. El gato: juego de multiplicación. (http://www.cientec.or.cr/matematica/gato.html; consultado 14 de mayo, 2010).

Centro Nacional de Ciencia y Tecnología. 2001b. Juegos cooperativos. (http://www.cientec.or.cr/matematica/juegos.html; consultado 14 de mayo, 2010).

Ferreiro, P. 2008. Indicaciones para estimular la lectura en la escuela. (http://www.surcultural.info/2008/05/indicacionespara-estimular-la-lectura-en-la-escuela/; consultado 14 de mayo, 2010).

Gómez, M. 2009. Elementos de estadística descriptiva. EUNED, San José, Costa Rica.

Hernández, O. 2009. Estadística elemental para Ciencias Sociales. Universidad de Costa Rica, San José, Costa Rica.

Jiménez, I. 1997. Informe de práctica docente supervisada III. Primeras experiencias prácticas en la labor docente. UNED. Diplomado en ciencias de la educación con énfasis en I y II ciclos. UNED, San José, Costa Rica.

Kelbe, E. 2003. Reflections on Special Programming for the Gifted Disadvantaged Students, p. 86-94, In J. Smutny (ed.). Designing and Developing Programs for Gifted Students. Corwin, California, Estados Unidos.

Leal, A. 2003. Formes de veure, formes d'entendre una historia: elements per elaborar una crítica. (http://www.aulamedia.org/aleal2.htm; consultado 18 de mayo, 2010)
Lowenfeld, V. \& W. Lambert. 1972. Desarrollo de la capacidad creadora. Kapelusz, Buenos Aires, Argentina.

Massip, A. 2004. Metodología para estimular en escolares de quinto y sexto grados la lectura creadora. Dirección provincial de educación de Sancti Spìritus. Tribunal de ciencias pedagógicas para las provincias centrales. Tesis en opción al grado científico de Doctora en Ciencias Pedagógicas. la Universidad de Ciencias Pedagógicas de Sancti Spiritus, Cuba.

Méndez, Z., A. Mainieri, A. Ortiz, P. Castro \& L. Paniagua. 1992. Estudio psicogenético del proceso de aprendizaje en niños talentosos de la Región de Turrialba. Mimeografiado, Instituto para el Mejoramiento de la Educación Costarricense (IIMEC), Universidad de Costa Rica, San José, Costa Rica.

Méndez, Z. \& Z. Pereira. 1985. Estudios psicogenéticos sobre el proceso de enseñanza-aprendizaje de la matemática., Instituto para el mejoramiento de la educación costarricense (IIMEC) Publicación interna. Universidad de Costa Rica. San José, Costa Rica.

Mirman, N. 2003. Identifying and Selecting Teachers, p. 39-47, In J. Smutny (ed.). Designing and Developing Programs for Gifted Students. Corwin, California, Estados Unidos.

Morales, K. 2008. Macroinvestigación: Identificación temprana con base empírica de niños y niñas entre 4 y 6 años con superdotación intelectual. Validación en Costa Rica. (http://www.uned.ac.cr/altadotacionytalentos/documentos/webldentificacionTemprana.pdf; consultado 14 de febrero del 2001)

Morales, K. 2009. Conceptualización docente acerca de las características, necesidades educativas especiales y posibles respuestas pedagógicas para los educandos con superdotación de Costa Rica. Cuadernos de investigación de la UNED. 1: 1-12.

Oviedo, J. \& Z. Méndez. 1984. Estudio de problemas aditivos. Memoria del Seminario sobre Creatividad y Talento. Mimeografiado. Universidad de Costa Rica, San José, Costa Rica.

Quirós, G. 2000. Guía metodológica para promover la lectura y la producción textual escrita en I ciclo de educación primaria. La aventura de leer y escribir. Tesis para optar por el grado de máster en planificación curricular Universidad de Costa Rica.

Renzulli, J. 2005. Applying Gifted Education Pedagogy to Total Talent Development for All Students. Theory into practice 44: 80-89.

Renzulli, J. 2008. La educación del sobredotado y el desarrollo del talento para todos. Revista de Psicología. 26: 24-42.

Reis, S. 2004. Series Introduction, p. 9-21, In J. Renzulli (ed.). Identification of Students for Gifted and Talented Programs. Corwin, California, Estados Unidos.

Rey, A. 1969. Epreuves d'automatismes intellectuels et scolaires. Delachaux et Niestlé. Neuchâtel, France. 
Smutny, J. \& C. Lind. 2003. From Needs and Goals to Program Organization: A Nuts-and-Bolts Guide, p. 7-29, In J. Smutny (ed.). Designing and Developing Programs for Gifted Students. Corwin, California, Estados Unidos.

Sternberg, R. \& P. Clinkenbeard. 1995. The triarchic model applied to identifying, teaching, and assessing gifted children. Roeper Review, 17: 255.
Valadez, M. 2004. Niñas, superdotación y contexto social. Educar 29: 53-58.

Van de Walle, J. 1993. Elementary school mathematics. Teaching developmentally. Longman, Nueva York, Estados Unidos.

Vergnaud, G. 1981. L'enfant, la mathématique et la reálité. Editions Peter Lang. Berne, Francia. 


\section{APÉNDICE 1}

\section{Cuestionario a docentes para la identificación de estudiantes con altas capacidades}

Lea cuidadosamente las siguientes características y anote en la hoja adjunta los nombres de sus estudiantes que de acuerdo a su conducta y desempeño académico las reflejen en su totalidad:

1. Que es el(a) mejor...

2. Que tiene mejor vocabulario...

3. Más creativo(a) y original...

4. Mayor capacidad de liderazgo...

5. Mejor orientado(a) científicamente...

6. Mejor pensamiento crítico...

7. Perturba más...

8. Más motivado(a)...

9. Del que los demás gustan más...

10. Más adelantado(a) en los estudios académicos...

11. Más brillante...

12. Cuyos padres se preocupan más por elevar su rendimiento académico...

13. Que tiene alguna habilidad especial...

14. Mejor representante del grupo...

15. Con dificultad de aprendizaje y considerado(a) inteligente...

16. Considerado(a) inteligente y con problemas emocionales...

17. Que aprende con rapidez (1-2 repeticiones para dominar)...

18. Formula preguntas complejas/profundas...

19. Construye ideas abstractas...

20 . Concluye con deducciones...

21. Es observador(a) preciso(a)...

22. Es sumamente autocrítico (a)...

23. Se distrae pero logra buenos resultados...

24. Tiene ideas descabelladas y sin sentidos...

25. Disfruta al aprender...

26. Demuestra fuertes sentimientos y opiniones... 


\section{APÉNDICE 2}

\section{Percepción de las maestras sobre el estudiante}

Maestra:

Sección:

Escuela:

Estudiante:

Por favor, marque a continuación con una equis $(X)$ en la casilla que corresponda según lo que usted ha observado sobre el estudiante.

\begin{tabular}{|l|l|l|l|l|}
\hline & Siempre & $\begin{array}{c}\text { Casi } \\
\text { Siempre }\end{array}$ & A veces & Nunca \\
\hline Es creativo y original & & & & \\
\hline Es líder de su grupo & & & & \\
\hline Está motivado para el estudio & & & & \\
\hline Tiene buen vocabulario & & & & \\
\hline Aprende con rapidez & & & & \\
\hline Pone atención en clase & & & & \\
\hline Formula preguntas en clase & & & & \\
\hline $\begin{array}{l}\text { Completa los trabajos en la } \\
\text { clase }\end{array}$ & & & & \\
\hline Termina las tareas para la casa & & & & \\
\hline
\end{tabular}

¿Se interesan los padres en el rendimiento académico de su hijo/a? ( )Sí ( )No

¿Tiene el estudiante alguna habilidad especial?

¿Presenta el estudiante algún problema de aprendizaje?

¿Presenta el estudiante algún tipo de problemas emocionales?

¿Cuál es su opinión sobre el estudiante? 
